\title{
High expression of CD52 mRNA predicts poor prognosis for cytogenetic normal acute myeloid Leukemia patients
}

\author{
Ping Cai \\ The First Affiliated Hospital of Soochow University \\ Wenzhi Cai \\ Southern Medical University Nanfang Hospital \\ Xiaoyu Xu \\ Southern Medical University Nanfang Hospital \\ Xiaofei Yang \\ Southern Medical University Nanfang Hospital \\ Yemin Wang \\ The First Affiliated Hospital of Soochow University

\section{Depei Wu} \\ The First Affiliated Hospital of Soochow University
}

Suning Chen ( $\square$ chensuning@suda.edu.cn)

Jiangsu Institute of Hematology, National Clinical Research Center for Hematologic Diseases, NHC Key Laboratory of Thrombosis and Hemostasis, The First Affiliated Hospital of Soochow University, Suzhou, China Collaborative Innovation Center of Hematology, https://orcid.org/0000-0001-6294-972X

\section{Research}

Keywords: Cytogenetic normal acute myeloid leukemia, CN-AML, CD52, Prognosis

Posted Date: March 27th, 2020

DOI: https://doi.org/10.21203/rs.2.22823/v2

License: () (1) This work is licensed under a Creative Commons Attribution 4.0 International License. Read Full License 


\section{Abstract}

Background: The prognosis of cytogenetic normal acute myeloid leukemia (CN-AML) varies. Finding new biomarkers affecting the prognosis of these patients may bring a new strategy for precise classification and treatment. CD52 plays a significant role in chronic lymphocytic leukemia (CLL). However, the potential role of CD52 in CN-AML remains largely elusive.

Methods: We analyzed the prognostic role of different expression levels of CD52 in 58 CN-AML from The Cancer Genome Atlas (TCGA) dataset and validated these results with $345 \mathrm{CN}-\mathrm{AML}$ patients from Gene Expression Omnibus (GEO) dataset.

Results: CN-AML patients with high CD52 mRNA expression had a poorer prognosis compared to low CD52 expression ( event-free survival [EFS], $\mathrm{P}=0.056$; overall survival [OS], $\mathrm{P}=0.043$; log-rank test) and the results was verified by GSE12417 (OS, P=0.020; log-rank test) and GSE71014 (OS, $\mathrm{P}=0.020$; log-rank test). Hematopoietic stem cell transplantation (HSCT) may improve prognosis of patients with CD52 high . Regression analysis shows that the expression level of CD52 (HR=1.503; 95\%Cl:1.158-1.949; $P=0.002)$ is a prognostic factor independent of age (HR=3.045; 95\%Cl:1.524-6.086; $P=0.002)$ and $F L T 3$ mutation status $(\mathrm{HR}=2.219 ; 95 \% \mathrm{Cl}: 1.123-4.382 ; \mathrm{P}=0.022)$. CD52 gene expression shows a predictive effect on EFS (1-year survival-area under the curve [AUC]:0.685, 2-year survival-AUC:0.752) and OS (1-year survival-AUC: 0.717, 2-year survival-AUC:0.770). Besides, we also found that there is a significant negative correlation between CD52 mRNA expression and DNA methylation. Accordingly, we speculated that CD52 DNA hypomethylation may responsible for the high level of CD52 mRNA. Functional enrichment analysis of differentially expressed genes in CD52 high and CD52 low suggests that adhesion molecule deregulation maybe also the potential pathological mechanism of CD52.

Conclusions: CD52 gene mRNA overexpression is an independent adverse prognostic factor for CN-AML. CD52 DNA hypomethylation may responsible for the high level of CD52 mRNA. Adhesion molecule deregulation maybe potential pathological mechanism of CD52. Whether CD52 monoclonal antibodies play a role in high risk patients need further research.

\section{Background}

Acute myeloid leukemia (AML) is a genetically heterogeneous malignant disease with adverse clinical outcome. The prognosis of these patients is mainly influenced by cytogenetic and molecular aberrations. Allogeneic hematopoietic stem cell transplantation (allo-HSCT) in the first complete remission (CR1) is recommended for high-risk AML patients, whereas data are less clear in the intermediate cytogenetic subgroup, particularly cytogenetic normal acute myeloid leukemia (CN-AML)(1). The prognosis of these patient can be further stratified according to different gene mutation such as FLT3, NPM1, CEBPA (2). In addition to genetic mutations, abnormal gene expression, such as NCALD, PDK2,PDK3, BAALC, CDKN1B, ERG, and MN1 etc. also used to predict the prognosis of CN-AML(3-5). Therefore, it is significant to identify new biomarkers to predict the prognosis of CN-AML, which may provide a new perspective to develop effective strategies to improve the prognosis of these patients.

CD52 (Campath-1) is a 21 to 28 kilodalton glycoprotein composed of 12 amino acid sequences, which is mainly expressed on normal and malignant B and T lymphocytes $(6,7)$ and some acute hematological diseases(8-10). It was reported that CD52 is an adverse predictor in cutaneous T-cell lymphoma (CTCL) and "double-hit" and "double-expressor" lymphomas $(11,12)$. CD52 expression on neoplastic stem cells (NSCs) was proved to be correlated with a poor survival in myelodysplastic syndromes (MDS) and AML patients with 5q- (13). As we know that the CD52 antigen is a glycoprotein anchored on the cell membrane, which may shed from cells as soluble CD52(14). Soluble CD52 antigen is also a significant survival predictor in CLL(15). CAMPATH-1H (alemtuzumab), CD52-specific monoclonal antibody (mAb), inducing cell lysis by activating complement and cell-mediated cytotoxicity, had been already approved for the treatment of relapsed or refractory B-cell chronic lymphocytic leukemia $(C L L)(16,17)$. Although targeting CD52 molecule was used as an approach to treat patients in clinics, the physiological and pathological significance and prognostic value of CD52 molecule is less understood so far, especially in CN-AML. Herein, we systemically evaluated the clinical significance of CD52 mRNA expression in CN-AML, which may provide new insights for prognostic stratification and individualized strategies.

\section{Patients And Methods}

\section{Data source and preprocessing}

A total of 58 de novo CN-AML patients with RNA-seq data which was showed with FPKM value (Fragments Per Kilobase Per Million Mapped Fragments) were detected from The Cancer Genome Atlas (TCGA; https://tcga-data.nci.nih.gov/) and relative expression values of mRNA was log-transformed using log2. The methylation data (Illumina Human Methylation 450k) was found in 48 of 58 patients.

Two independent expression data of 242 CN-AML patients in GSE12417 and 104 CN-AML in GSE71014 were downloaded from the National Center of Biotechnology Information-Gene Expression Omnibus (NCBI-GEO) database (https://www.ncbi.nlm.nih.gov/geo). Expression values were all normalized in GEO arrays. Gene expression in GSE12417 was performed by using the Affymetrix Human Genome U133A Array (GPL96; N=163) and Affymetrix Human Genome U133 Plus 2.0 Array (GPL570; N=79). We had corrected batch effect between these two platforms using the sav package in R (version 3.6.1; http://www.r-project.org). Microarray data in GSE71014 was done with Illumina HumanHT-12 V4.0 expression beadchip (GPL10558; N=103). All mRNA expression values were converted by log2.

\section{Statistical and bioinformatics analysis}

Considering of clinical significance, age was transformed into categorical variables. Pearson Chi-square analysis or Fisher exact test was applied for the comparison of categorical variables. Univariate and multivariate Cox regression were performed to assess the proportional hazard. Prognostic impact of CD52 gene expression was analyzed through Kaplan-Meier analysis (Log-rank test). Overall survival (OS) is the time from AML diagnosis until death from any 
cause or last clinical follow-up. Event-free survival (EFS) is the time from diagnosis to removal from the study due to death, relapse, failure to achieve complete remission (CR), or censored at the last follow-up. Area under the receiver operating characteristic curve (AUC-ROC) analysis was performed with the timeROC package. Comparisons between two different subgroups of continuous parameters were made using Kruskal-Wallis test. The Pearson correlation test was used to measure associations among continuous variables. A two-tailed $P$ value of less than 0.05 was considered statistically significant for above analyses. Different gene expression between two group was performed with EdgeR package using the raw read counts. False discovery rate (FDR)<0.05 and |log2 fold change (FC)| > 1 was considered to be significantly different in gene expression. Enrichment analysis of Gene ontology (GO) function and Kyoto encyclopedia of genes and genomes (KEGG) pathway for different expression genes were performed by "org.Hs.eg.db", "clusterProfiler", "ggplot2" and "enrichplot" packages and $\mathrm{P}$ value $<0.05$ was regarded as statistically significant differences.

\section{Results}

\section{Baseline patient characteristics}

A total of $58 \mathrm{CN}$-AML patients were included in the research. Thirty patients received chemotherapy alone, and the remaining twenty-eight patients were proceeded with allogeneic hematopoietic stem cell transplantation. We divided the patients into high expression group and low expression group according to the CD52 gene median FPKM value. The clinical and molecular characteristics between the two groups were compared (Table 1). The median ages of CD52 ${ }^{\text {high }}$ and $C D 52^{\text {low }}$ were 51 years (range, 21-75 years) and 63 years (range, 21-88 years), respectively. The patients in CD52 ${ }^{\text {high }}$ cohort were older than low expression cohort $(P=0.019)$ and the mutation ratio of $D N M T 3 A$ was higher in the latter group $(P=0.014)$. There were no significant differences between the two groups in sex, race, FAB classification, chemotherapy, transplant, genetic mutations (NPM1, FLT3, IDH1, IDH2, RUNX1) (all P values > 0.05). The baseline characteristics patients in GSE12417 and GSE71014 had already described in previous studies(18, 19).

\section{High expression of CD52 is a poor prognostic marker for CN-AML patients}

In order to evaluate the impact of CD52 expression on the survival CN-AML of patients, we employed the Kaplan-Meier method and log-rank test in 58 CNAML patients from TCGA dataset. Results showed that $C D 52^{\text {high }}$ group had shorter EFS (Fig. $\left.1 \mathbf{a} ; \mathrm{P}=0.056\right)$ and $0 \mathrm{~S}$ (Fig. $1 \mathbf{b}$; $\mathrm{P}=0.043$ ). To validate the results, we analyzed CD52 gene expression profile of 242 CN-AML in GSE12417 and 103 CN-AML in GSE71014. The patients with high level of CD52 expression showed a poor OS in independent cohorts (Fig. $1 \mathrm{c}-\mathrm{d} ; \mathrm{P}=0.007, \mathrm{P}=0.020$, respectively).

$58 \mathrm{CN}-A M L$ were divided into the chemotherapy-only group $(n=30)$ and allo-HSCT group $(n=28)$. Kaplan-Meier survival curves suggested that CD 52 high was an adverse factor for chemotherapy group (EFS, $p=0.041$; OS, $p=0.013$; Fig. $1 \mathrm{e}-\mathrm{f}$ ), whereas the expression level of CD 52 played a small role in the survival of HSCT group (EFS, $p=0.3647$; OS, $p=0.4812$; Additional file: Fig.S1 a-b). HSCT may prolong overall survival of CD 52 $2^{\text {high }}$ ( $p=0.0795$ ) patients in some extent but $C D 52^{\text {low }}(p=0.4812)$ (Additional file: Fig.S1 $\left.c-d\right)$. CD $52^{\text {low }}$ patients could receive a long-term remission with standard intensive chemotherapy and HSCT is adviced for $\mathrm{CD} 52^{\text {high }}$ patients.

\section{CD52 expression is an independent risk factor for prognosis in patients with CN-AML}

Univariate and Multivariate cox regression analysis were implemented to evaluate the prognostic value of clinical and biological variables. We analyzed sex, age, CD52 gene expression and gene mutations, such as FLT3, NPM1, IDH1, IDH2, RUNX1, DNMT3A in univariate cox regression analysis. Univariate analysis showed that CD52 gene expression level was associated with shorter OS (Fig.2a; HR=1.465; $P=0.001$ ) and EFS (Fig.2b; HR=1.29; $P=0.036)$. Besides, FLT3 mutation also contributed to worse OS $(H R=1.903 ; P=0.049)$ and $E F S(H R=2.084 ; P=0.030)$. Age only affected $O S(H R=2.466 ; P=0.007)$ rather than $E F S$ $(P=0.215)$. Other variables had no significance for the prognosis of $C N-A M L$ patients (all $P$ values $>0.05)$. Therefore, age, FLT3 genes mutation and CD52 gene expression levels were chosen for multivariate cox regression analysis. Age (HR=3.045; 95\% confidence interval [Cl]:1.524-6.086; $P=0.002)$, FLT3 mutation $(\mathrm{HR}=2.219 ; 95 \% \mathrm{Cl}: 1.123-4.382 ; \mathrm{P}=0.022)$, CD52 gene expression level (HR=1.503; 95\% Cl: $1.158-1.949 ; \mathrm{P}=0.002)$ were independent risk factors of $0 \mathrm{~S}$ for $\mathrm{CN}$ AML patients (Fig.2c). FLT3 was the only factor that affect EFS (Fig.2d; HR=2.318, 95\%Cl: 1.138-4.722, P=0.021) in CN-AML patients.

Area under the receiver operating characteristic curve (AUC-ROC) analysis was performed to assess the prediction accuracy of EFS and OS with CD52 gene expression. CD52 gene expression showed a predictive effect on EFS (Fig.3a; 1year survival-AUC:0.685, 2year survival-AUC:0.752) and OS (Fig.3b;1year survival-AUC: $0.717,2$ year survival-AUC:0.770).

\section{Correlation of CD52 mRNA expression with other biomarkers in CN-AML patients}

We analyzed the relationship of mRNA expression between CD52 and the genes mutations that were reported to affect the prognosis of CN-AML patients. The mutation of CEBPA was associated with lower levels of CD52 mRNA (Fig.4a, $p=0.001$ ), while the mutation of DNMT3A tended to show higher levels of CD52 (Fig.4b, $p=0.004)$. The gene mutation status, such as FLT3 ( $p=0.188)$, NPM1 ( $p=0.839)$, IDH1 ( $p=0.125)$, IDH2 ( $p=0.179)$, RUNX1 ( $p=0.818)$, showed no relationships with the expression level of CD52. Besides, we analyzed the relationship between the expression of CD52 and genes that affecting prognosis of CN-AML patients in previous studies, such as WT1(20), EVI1(21), FLT3(22), MN1(5, 23), ERG(5, 24), ID1(25), CDKN1B(5) and BAALC(5). There was no correlation between CD52 and gene above (Fig.4c; all $P$ values $>0.05$ ). CD52 play its' role independent of gene above in CN-AML. 
DNA methylation is the common factor associated with abnormal gene expression. We evaluated the correlations among CD52 gene mRNA expression and DNA methylation (average $\beta$-values). There was a moderate, significant, inverse correlation between DNA methylation and average gene expression (Fig.4d; r=-0.683, $\left.P=4.234 \times 10^{-6}\right)$. CD52 gene DNA CpG sites, such as cg16068833 $\left(r=-0.665 ; p=1.178 \times 10^{-6}\right), c g 19677267\left(r=-0.607 ; p=1.607 \times 10^{-5}\right)$, $\operatorname{cg} 19743891\left(r=-0.648 ; p=2.642 \times 10^{-6}\right)$ also show a correlation with gene expression, which were not show here.

\section{Functional annotation and pathway enrichment of differentially expressed genes (DEGs)}

To gain insights into the biological function of CD52, we analyzed different gene expression in CD $52^{\text {high }}$ group and CD $52^{\text {low }}$ group. A total of 933 differentially expressed genes had been found (214 downregulated genes; 719 downregulated genes). The differentially expressed genes between two groups has been shown in volcano plot (Fig.4e). Go and KEGG functional annotation analysis has been shown in Fig.4f-g. GO analysis found that T cell activation is important $\mathrm{GO}$ category (32 gene; $\mathrm{P}=0.0005)$ in the biological process $(\mathrm{BP})$ ontology. In cellular component $(\mathrm{CC})$ ontology, the most significant $\mathrm{GO}$ category is $\mathrm{T}$ cell receptor complex (7 gene; $\mathrm{P}=0.000061)$. In molecular function (MF) ontology, the major histocompatibility complex (MHC) protein binding is the most important $\mathrm{GO}$ category ( 9 gene; $\mathrm{P}=0.0003$ ). KEGG analysis also show that differentially expressed proteins mainly enriched in $\mathrm{T}$ cell receptor signaling pathway. In addition to T cell activation-related pathways, some DEGs are enriched in leukocyte cell-cell adhesion and regulation of leukocyte cell-cell adhesion (Table2).

\section{Discussion}

The prognostic value of CD52 at protein level has been recognized in the CLL and some malignant lymphomas(12, 26). CD52 molecules or CD52+ microvesicles in plasma all play a role in disease prognosis $(12,15,26,27)$. Katharina et al. found that CD52 expression on NSCs was correlated with a poor survival in MDS and AML patients with 5q- (13). The majority of CD52 studies were investigated at protein level using flow cytometry technology or ELISA analyses, while the prognostic value of CD52 at mRNA level is little known, especially in the CN-AML. A research leaded by Lin et al mentioned the prognostic value of CD52 in CN-AML (28). However, the research focused on MPG6(6-Membrane Protein Gene) and CD52 only a part of MPG6. Our study performed a comprehensive bioinformatics analysis of CD52 in CN-AML. In addition to the prognostic value of CD52, We also analyzed possible pathological mechanism of CD52.

CD52 mRNA is a prognostic marker for CN-AML patients. However, it is not clear whether the prognostic value is independent or based on other prognostic factors. We analyzed the correlation of CD52 mRNA level with age, gene mutation and other biomarkers, which was reported as prognostic markers in intermediate-risk AML. There is no correlation between age and CD52 mRNA expression level, which may eliminate abnormal gene expression related to aging. CN-AML patients with CEBPA mutation shows a relatively good prognosis, while DNMT3A presents adverse(29, 30). Mutation of CEBPA and DNMT3A genes are associated with lower and higher expression of CD52 mRNA respectively. The association verified the prognostic value of the CD52 gene. However, whether the pathogenic effects of mutation and CD52 were independent or interrelated remains to be verified. There is a significant negative correlation between gene expression and DNA methylation. The result suggests that CD52 gene hypomethylation may responsible for the overexpression of CD52 mRNA in CN-AML. Obviously, further studies are needed to confirm the direct connections of CD52 mRNA expression with methylation.

CD52 is an important immune regulator on T-cell activation as previous reports, which can modulate T-cell activation either by its intracellular signal pathways or by the interaction of soluble CD52 and Siglec-10 expressing on T cells(31,32). Functional enrichment analysis of differential expression genes in CD52 $2^{\text {high }}$ and $C D 52^{\text {low }}$ found that DEGs were significantly enriched in terms of T cell activation, which is consistent with previous reports. In addition to $T$ cell activation-related pathways, some DEGs enriched in leukocyte cell-cell adhesion, regulation of leukocyte cell-cell adhesion, positive regulation of cell-cell adhesion and cell adhesion molecules (CAMs). Many oncogenes, such as BCR-ABL1, RUNX1-ETO, MLL-AF6 etc., directly regulate the activity of adhesion molecules to control malignant hematopoietic progenitor cell (28). We speculated that adhesion molecule deregulation maybe the potential pathological mechanism of CD52. However, whether CD52 play its role in this manner needs more experiments to confirm.

CD52 monoclonal antibody had already been approved for the treatment of relapsed or refractory CLL. The use of CD52 mAb in the AML is rarely reported. Raoul et al. found that Single-agent alemtuzumab have limited activity in CD52-positive recurrent or refractory acute leukemia(33). Our research found CD52 ${ }^{\text {high }}$ was an adverse prognostic factor for CN-AML. However, whether patients with high CD52 expression will benefit from CD52 mAb in combination with chemotherapy needs further research.

The limitation of the research also need mention. The patients in our research are from three different datasets and received different chemotherapy strategies, which may influence the result to some extent. Besides, bioinformatics analysis only provided possible clues to the pathological mechanism of CD52, and more experimental data are needed to verify our guess.

\section{Conclusion}

CD52 gene mRNA overexpression is an adverse prognostic factor for CN-AML patients. Possible underlying pathological mechanism needs further verification

\section{List Of Abbreviations}

CN-AML: cytogenetic normal acute myeloid leukemia. AML: acute myeloid leukemia. CLL: chronic lymphocytic leukemia. TCGA: The Cancer Genome Atlas. GEO: Gene Expression Omnibus. EFS: event-free survival. OS: overall survival. Allo-HSCT: Allogeneic hematopoietic stem cell transplantation. AUC: area under the curve. AUC-ROC: Area under the receiver operating characteristic curve. CR1: first complete remission. CTCL: cutaneous T-cell lymphoma. NSCs: neoplastic 
stem cells. MDS: myelodysplastic syndromes. mAb: monoclonal antibody. NCBI-GEO: National Center of Biotechnology Information-Gene Expression Omnibus. DEGs: differentially expressed genes. FDR: False discovery rate. FC: fold change GO: Enrichment analysis of Gene ontology. KEGG: Kyoto encyclopedia of genes and genomes.

\section{Declarations}

\section{Ethics approval and consent to participate}

The written informed consent of all patients in this study was consistent with the Helsinki Declaration.

\section{Consent for publication}

Not applicable.

\section{Availability of data and materials}

The datasets analyzed in the study are available from the corresponding author.

\section{Competing interests}

All authors declare that they have no competing interests.

\section{Funding}

This work was supported by grants from the Natural Science Foundation of China (81600116, 81570139, 81700140), the nature science Foundation of the Jiangsu Higher Education Institutions of China (18KJA320005), the Innovation Capability Development Project of Jiangsu Province (BM2015004), the Jiangsu Provincial Key Medical Center (YXZXA2016002), the Priority Academic Program Development of Jiangsu Higher Education Institutions, and the Key research and development plan of Jiangsu Province(BE2019655).

\section{Authors' contributions}

$\mathrm{CP}$ and $\mathrm{CSN}$ conceived the projects. CP, CWZ and XXY analyzed the data. CP, CWZ, XXY, YXF, WYM, ZMJ, WDP and CSN contributed towards the interpretation of the data. All authors read and approved the final manuscript.

\section{Acknowledgements}

The data is derived from TCGA dataset and GEO dataset (GSE12417, GSE71014)

\section{Additional File Information}

Additional file1: Figure S1.a-b: Kaplan-Meier survival curves of OS( $p=0.3647)$ and EFS $(p=0.4812)$ in hematopoietic stem cell transplantation cohort; $c-d$ : Kaplan-Meier survival curves of OS in $\operatorname{CD}_{5} 2^{\text {high }}(p=0.0795)$ and $C_{5} 2^{\text {low }}(p=0.4812)$ group.

\section{Tables}




\begin{tabular}{|c|c|c|c|}
\hline \multirow[t]{2}{*}{ Characteristics } & \multicolumn{2}{|l|}{ TCGA } & \multirow[t]{2}{*}{ P-value } \\
\hline & CD52-low & CD52-high & \\
\hline $\mathrm{N}$ & 29 & 29 & \\
\hline Sex $(\%)$ & & & 0.066 \\
\hline Female & 11(18.97\%) & $18(31.03 \%)$ & \\
\hline Male & $18(31.03 \%)$ & $11(18.91 \%)$ & \\
\hline Age(median/range) & $51(21-75)$ & $63(21-88)$ & 0.019 \\
\hline$\geq 65$ & $4(6.90 \%)$ & $12(20.69 \%)$ & \\
\hline$<65$ & $25(43.10 \%)$ & $17(29.31 \%)$ & \\
\hline Race (\%) & & & 0.769 \\
\hline Black & $23(39.66 \%)$ & $20(34.48 \%)$ & \\
\hline White & $2(3.45 \%)$ & $2(3.45)$ & \\
\hline Unknown & $5(8.63 \%)$ & $7(12.07)$ & \\
\hline FAB (\%) & & & 0.136 \\
\hline M1 & $1(1.72 \%)$ & $2(3.45 \%)$ & \\
\hline M2 & $13(22.41 \%)$ & $5(8.62 \%)$ & \\
\hline M4 & $8(13.79 \%)$ & $8(13.79 \%)$ & \\
\hline M5 & $3(5.17 \%)$ & $9(15.52 \%)$ & \\
\hline M6 & $4(6.9 \%)$ & $5(8.62 \%)$ & \\
\hline Treatment (\%) & & & 0.115 \\
\hline Chemotherapy & $12(20.69 \%)$ & 18(31.03\%) & \\
\hline HSCT & $17(29.31 \%)$ & 11(18.97\%) & \\
\hline Relapse (\%) & & & 0.785 \\
\hline Yes & $18(31.03 \%)$ & $19(32.76 \%)$ & \\
\hline NO & 11(18.97\%) & $10(17.24 \%)$ & \\
\hline CEBPA (\%) & & & 0.004 \\
\hline Mutation & $8(13.79 \%)$ & $0(0 \%)$ & \\
\hline WT & $21(36.21 \%)$ & $29(50 \%)$ & \\
\hline FLT3-ITD (\%) & & & 0.588 \\
\hline Mutation & $10(17.24 \%)$ & $12(20.31 \%)$ & \\
\hline WT & $19(32.76 \%)$ & $17(29.31)$ & \\
\hline NPM1 (\%) & & & 0.293 \\
\hline Mutation & $17(29.31 \%)$ & $13(22.41 \%)$ & \\
\hline WT & $12(20.69 \%)$ & $16(27.59 \%)$ & \\
\hline IDH1 (\%) & & & 0.253 \\
\hline Mutation & $6(10.34 \%)$ & $2(3.45 \%)$ & \\
\hline WT & 23(39.66\%) & $27(46.55 \%)$ & \\
\hline IDH2 (\%) & & & 0.145 \\
\hline Mutation & $2(3.45 \%)$ & $7(12.07 \%)$ & \\
\hline WT & $27(46.55 \%)$ & $23(39.66 \%)$ & \\
\hline RUNX1 (\%) & & & 0.414 \\
\hline Mutation & $2(3.45 \%)$ & $4(6.9 \%)$ & \\
\hline WT & $27(46.55 \%)$ & $23(39.66 \%)$ & \\
\hline
\end{tabular}




\begin{tabular}{|cllc|}
\hline Characteristics & \multicolumn{2}{l}{ TCGA } & \multirow{2}{*}{ P-value } \\
\cline { 2 - 3 } & CD52-low & CD52-high & \\
\hline DNMT3A (\%) & & & 0.014 \\
\hline Mutation & $6(10.34 \%)$ & $15(25.86 \%)$ & \\
WT & $23(39.66 \%)$ & $14(24.14 \%)$ & \\
\hline
\end{tabular}

Table 1 Pretreatment characteristics of 58 CN-AML patients CD52lowand CD52high cohort

\begin{tabular}{|c|c|c|c|c|}
\hline ID & Description & p.adjust & geneID & Count \\
\hline GO:0007159 & $\begin{array}{l}\text { leukocyte } \\
\text { cell-cell } \\
\text { adhesion }\end{array}$ & 0.0033037 & $\begin{array}{l}\text { DPP4/ADTRP/ICOS/CD6/ETS1/CD3E/IL7R/SKAP1/CD40LG/LCK/GATA3/TIGIT/ } \\
\text { LAG3/TBX21/IFNG/BMP7/EFNB3/CTLA4/CCR7/SIRPG/CD28/SELP/HES1/IDO1 }\end{array}$ & 24 \\
\hline GO:0022409 & $\begin{array}{l}\text { positive } \\
\text { regulation } \\
\text { of cell-cell } \\
\text { adhesion }\end{array}$ & 0.0043429 & $\begin{array}{l}\text { DPP4/ICOS/CD6/ETS1/CD3E/IL7R/SKAP1/CD40LG/LCK/GATA3/IFNG/ } \\
\text { BMP7/EFNB3/CTLA4/CCR7/SIRPG/CD28/ANK3/WNT5A/HES1 }\end{array}$ & 20 \\
\hline GO:1903037 & $\begin{array}{l}\text { regulation } \\
\text { of } \\
\text { leukocyte } \\
\text { cell-cell } \\
\text { adhesion }\end{array}$ & 0.0043429 & $\begin{array}{l}\text { DPP4/ADTRP/ICOS/CD6/ETS1/CD3E/IL7R/SKAP1/CD40LG/LCK/GATA3/ } \\
\text { TIGIT/LAG3/TBX21/IFNG/EFNB3/CTLA4/CCR7/SIRPG/CD28/HES1/IDO1 }\end{array}$ & 22 \\
\hline hsa04514 & $\begin{array}{l}\text { Cell } \\
\text { adhesion } \\
\text { molecules } \\
\text { (CAMs) }\end{array}$ & 0.0249930 & $\begin{array}{l}\text { CD226/ICOS/CD2/CD6/CD40LG/TIGIT/CADM1/CD8A/CTLA4/ } \\
\text { CD28/SELP/CD8B }\end{array}$ & 12 \\
\hline
\end{tabular}

Table 2 Enrichment analysis of Gene ontology (GO) function and Kyoto encyclopedia of genes and genomes (KEGG) pathway

\section{References}

1. Schmid C, Labopin M, Socie G, Daguindau E, Volin L, Huynh A, et al. Outcome of patients with distinct molecular genotypes and cytogenetically normal AML after allogeneic transplantation. Blood. 2015;126(17):2062-9.

2. Gregory TK, Wald D, Chen Y, Vermaat JM, Xiong Y, Tse W. Molecular prognostic markers for adult acute myeloid leukemia with normal cytogenetics. Journal of hematology \&amp; oncology. 2009;2:23.

3. Song Y, Zhang W, He X, Liu X, Yang P, Wang J, et al. High NCALD expression predicts poor prognosis of cytogenetic normal acute myeloid leukemia. Journal of translational medicine. 2019;17(1):166.

4. Fu L, Fu H, Tian L, Xu K, Hu K, Wang J, et al. High expression of RUNX1 is associated with poorer outcomes in cytogenetically normal acute myeloid leukemia. Oncotarget. 2016;7(13):15828-39.

5. Haferlach C, Kern W, Schindela S, Kohlmann A, Alpermann T, Schnittger S, et al. Gene expression of BAALC, CDKN1B, ERG, and MN1 adds independent prognostic information to cytogenetics and molecular mutations in adult acute myeloid leukemia. Genes, chromosomes \&amp; cancer. 2012;51(3):25765.

6. Xia MQ, Tone M, Packman L, Hale G, Waldmann H. Characterization of the CAMPATH-1 (CDw52) antigen: biochemical analysis and cDNA cloning reveal an unusually small peptide backbone. European journal of immunology. 1991;21(7):1677-84.

7. Xia MQ, Hale G, Lifely MR, Ferguson MA, Campbell D, Packman L, et al. Structure of the CAMPATH-1 antigen, a glycosylphosphatidylinositol-anchored glycoprotein which is an exceptionally good target for complement lysis. The Biochemical journal. 1993;293 (Pt 3):633-40.

8. Schub N, Gunther A, Schrauder A, Claviez A, Ehlert C, Gramatzki M, et al. Therapy of steroid-refractory acute GVHD with CD52 antibody alemtuzumab is effective. Bone marrow transplantation. 2011;46(1):143-7.

9. Hoermann G, Blatt K, Greiner G, Putz EM, Berger A, Herrmann H, et al. CD52 is a molecular target in advanced systemic mastocytosis. FASEB journal : official publication of the Federation of American Societies for Experimental Biology. 2014;28(8):3540-51.

10. Cohen JA, Coles AJ, Arnold DL, Confavreux C, Fox EJ, Hartung HP, et al. Alemtuzumab versus interferon beta 1a as first-line treatment for patients with relapsing-remitting multiple sclerosis: a randomised controlled phase 3 trial. Lancet (London, England). 2012;380(9856):1819-28.

11. Lefrancois P, Xie P, Wang L, Tetzlaff MT, Moreau L, Watters AK, et al. Gene expression profiling and immune cell-type deconvolution highlight robust disease progression and survival markers in multiple cohorts of CTCL patients. Oncoimmunology. 2018;7(8):e1467856.

12. Craig JW, Mina MJ, Crombie JL, LaCasce AS, Weinstock DM, Pinkus GS, et al. Assessment of CD52 expression in "double-hit" and "double-expressor" lymphomas: Implications for clinical trial eligibility. PloS one. 2018;13(7):e0199708.

13. Blatt K, Herrmann H, Hoermann G, Willmann M, Cerny-Reiterer S, Sadovnik I, et al. Identification of campath-1 (CD52) as novel drug target in neoplastic stem cells in 5q-patients with MDS and AML. Clinical cancer research : an official journal of the American Association for Cancer Research. 
2014;20(13):3589-602.

14. Albitar M, Do KA, Johnson MM, Giles FJ, Jilani I, O'Brien S, et al. Free circulating soluble CD52 as a tumor marker in chronic lymphocytic leukemia and its implication in therapy with anti-CD52 antibodies. Cancer. 2004;101(5):999-1008.

15. Vojdeman FJ, Herman SEM, Kirkby N, Wiestner A, van T' Veer MB, Tjonnfjord GE, et al. Soluble CD52 is an indicator of disease activity in chronic lymphocytic leukemia. Leukemia \&amp; lymphoma. 2017;58(10):2356-62.

16. Keating MJ, Flinn I, Jain V, Binet JL, Hillmen P, Byrd J, et al. Therapeutic role of alemtuzumab (Campath-1H) in patients who have failed fludarabine: results of a large international study. Blood. 2002;99(10):3554-61.

17. Stilgenbauer S, Dohner $\mathrm{H}$. Campath-1 $\mathrm{H}$-induced complete remission of chronic lymphocytic leukemia despite p53 gene mutation and resistance to chemotherapy. The New England journal of medicine. 2002;347(6):452-3.

18. Metzeler KH, Hummel M, Bloomfield CD, Spiekermann K, Braess J, Sauerland MC, et al. An 86-probe-set gene-expression signature predicts survival in cytogenetically normal acute myeloid leukemia. Blood. 2008;112(10):4193-201.

19. Chuang MK, Chiu YC, Chou WC, Hou HA, Tseng MH, Kuo YY, et al. An mRNA expression signature for prognostication in de novo acute myeloid leukemia patients with normal karyotype. Oncotarget. 2015;6(36):39098-110.

20. Schmid D, Heinze G, Linnerth B, Tisljar K, Kusec R, Geissler K, et al. Prognostic significance of WT1 gene expression at diagnosis in adult de novo acute myeloid leukemia. Leukemia. 1997;11(5):639-43.

21. Barjesteh van Waalwijk van Doorn-Khosrovani S, Erpelinck C, van Putten WL, Valk PJ, van der Poel-van de Luytgaarde S, Hack R, et al. High EVI1 expression predicts poor survival in acute myeloid leukemia: a study of 319 de novo AML patients. Blood. 2003;101(3):837-45.

22. Ozeki K, Kiyoi H, Hirose Y, Iwai M, Ninomiya M, Kodera Y, et al. Biologic and clinical significance of the FLT3 transcript level in acute myeloid leukemia. Blood. 2004;103(5):1901-8.

23. Langer C, Marcucci G, Holland KB, Radmacher MD, Maharry K, Paschka P, et al. Prognostic importance of MN1 transcript levels, and biologic insights from MN1-associated gene and microRNA expression signatures in cytogenetically normal acute myeloid leukemia: a cancer and leukemia group B study. Journal of clinical oncology : official journal of the American Society of Clinical Oncology. 2009;27(19):3198-204.

24. Metzeler KH, Dufour A, Benthaus T, Hummel M, Sauerland MC, Heinecke A, et al. ERG expression is an independent prognostic factor and allows refined risk stratification in cytogenetically normal acute myeloid leukemia: a comprehensive analysis of ERG, MN1, and BAALC transcript levels using oligonucleotide microarrays. Journal of clinical oncology : official journal of the American Society of Clinical Oncology. 2009;27(30):5031-8.

25. Marcucci G, Maharry K, Wu YZ, Radmacher MD, Mrozek K, Margeson D, et al. IDH1 and IDH2 gene mutations identify novel molecular subsets within de novo cytogenetically normal acute myeloid leukemia: a Cancer and Leukemia Group B study. Journal of clinical oncology : official journal of the American Society of Clinical Oncology. 2010;28(14):2348-55.

26. Alatrash G, Albitar M, O'Brien S, Wang X, Manshouri T, Faderl S, et al. Circulating CD 52 and CD20 levels at end of treatment predict for progression and survival in patients with chronic lymphocytic leukaemia treated with fludarabine, cyclophosphamide and rituximab (FCR). British journal of haematology. 2010;148(3):386-93.

27. Boysen J, Nelson M, Magzoub G, Maiti GP, Sinha S, Goswami M, et al. Dynamics of microvesicle generation in B-cell chronic lymphocytic leukemia: implication in disease progression. Leukemia. 2017;31(2):350-60.

28. Lin SY, Miao YR, Hu FF, Hu H, Zhang Q, Li Q, et al. A 6-Membrane Protein Gene score for prognostic prediction of cytogenetically normal acute myeloid leukemia in multiple cohorts. Journal of Cancer. 2020;11(1):251-9.

29. Dohner H, Estey E, Grimwade D, Amadori S, Appelbaum FR, Buchner T, et al. Diagnosis and management of AML in adults: 2017 ELN recommendations from an international expert panel. Blood. 2017;129(4):424-47.

30. Marcucci G, Metzeler KH, Schwind S, Becker H, Maharry K, Mrozek K, et al. Age-related prognostic impact of different types of DNMT3A mutations in adults with primary cytogenetically normal acute myeloid leukemia. Journal of clinical oncology : official journal of the American Society of Clinical Oncology. 2012;30(7):742-50.

31. Bandala-Sanchez E, N GB, Goddard-Borger ED, Ngui K, Naselli G, Stone NL, et al. CD52 glycan binds the proinflammatory B box of HMGB1 to engage the Siglec-10 receptor and suppress human T cell function. Proceedings of the National Academy of Sciences of the United States of America. 2018;115(30):7783-8.

32. Zhao Y, Su H, Shen X, Du J, Zhang X, Zhao Y. The immunological function of CD52 and its targeting in organ transplantation. Inflammation research : official journal of the European Histamine Research Society [et al]. 2017;66(7):571-8.

33. Tibes R, Keating MJ, Ferrajoli A, Wierda W, Ravandi F, Garcia-Manero G, et al. Activity of alemtuzumab in patients with CD52-positive acute leukemia. Cancer. 2006;106(12):2645-51.

\section{Figures}




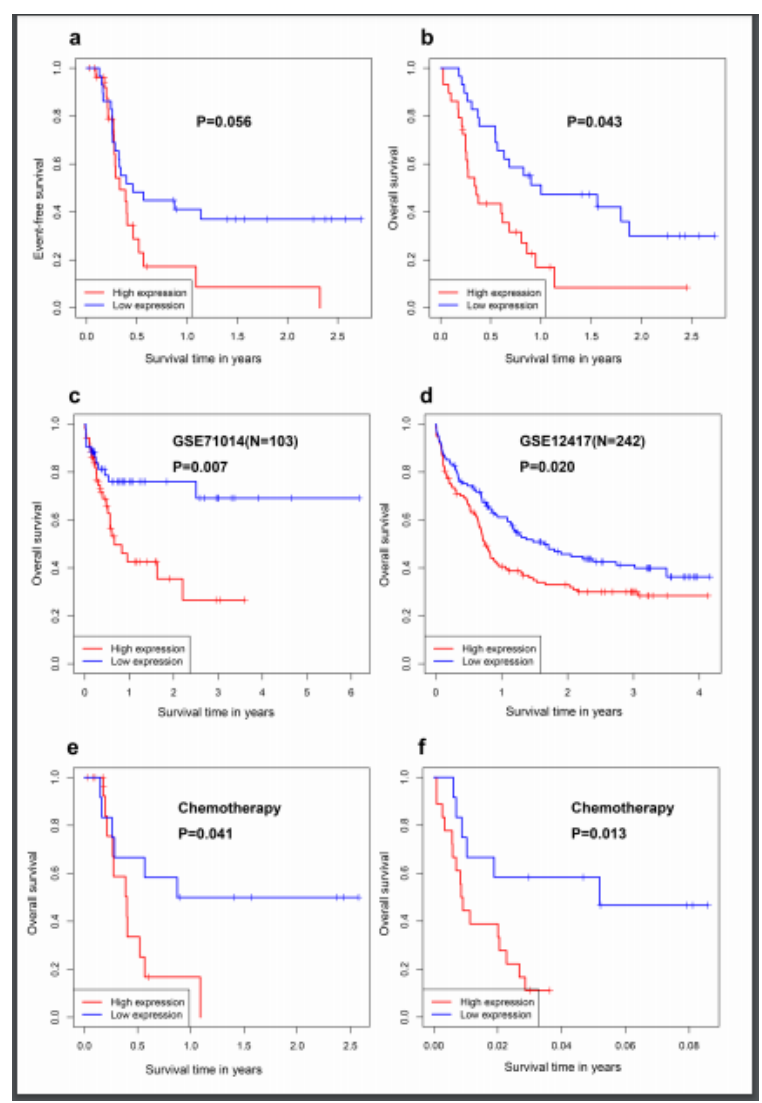

Figure 1

CD52 expression predicts survival of CN-AML patients. a-b: Kaplan-Meier survival curves of event-free survival (EFS, $P=0.0563)$ and overall survival (OS, $\mathrm{P}=0.0433)$ in different CD52 expression groups; $\mathrm{C}-\mathrm{d}$ : Kaplan-Meier survival curves of OS in GSE71014 (P=0.007) and GSE12417 (P=0.020); e-f: Kaplan-Meier survival curves of OS $(P=0.041)$ and $E F S(P=0.0133)$ in chemotherapy group.

\section{Univariate cox regression}

a

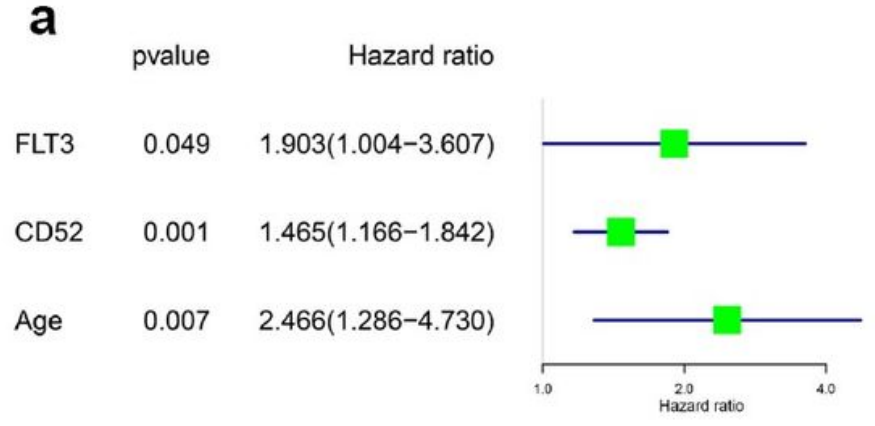

b

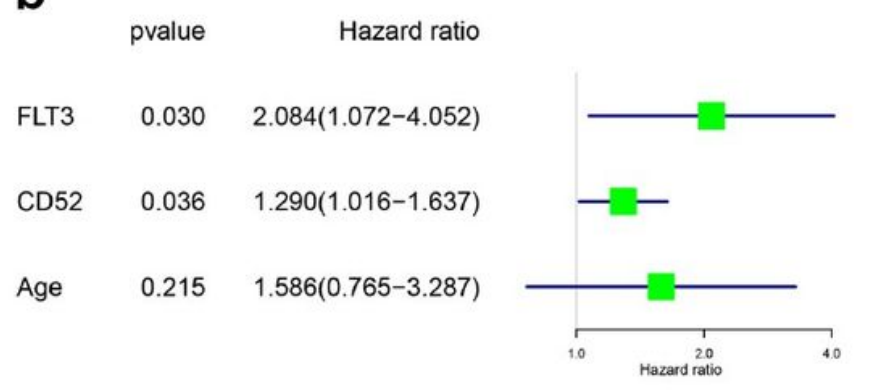

\section{Multivariate cox regression}

C

pvalue Hazard ratio

FLT3 $\quad 0.022 \quad 2.219(1.123-4.382)$

CD52 $0.002 \quad 1.503(1.158-1.949)$

Age $\quad 0.002 \quad 3.045(1.524-6.086)$

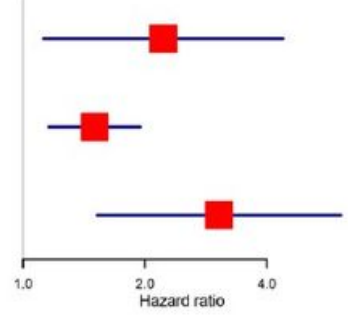

d

pvalue

Hazard ratio

FLT3 $\quad 0.021 \quad 2.318(1.138-4.722)$

CD52 $\quad 0.067 \quad 1.280(0.982-1.669)$

Age $\quad 0.070 \quad 2.052(0.942-4.471)$

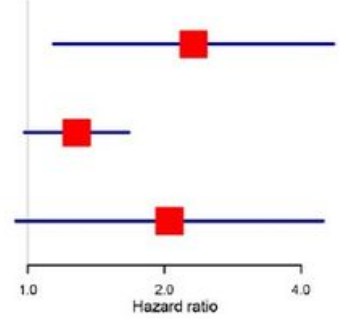

Figure 2 
CD52 expression is an independent risk factor of CN-AML patients. Univariate and multivariate analysis for OS (a, c) and EFS (b, d).

a

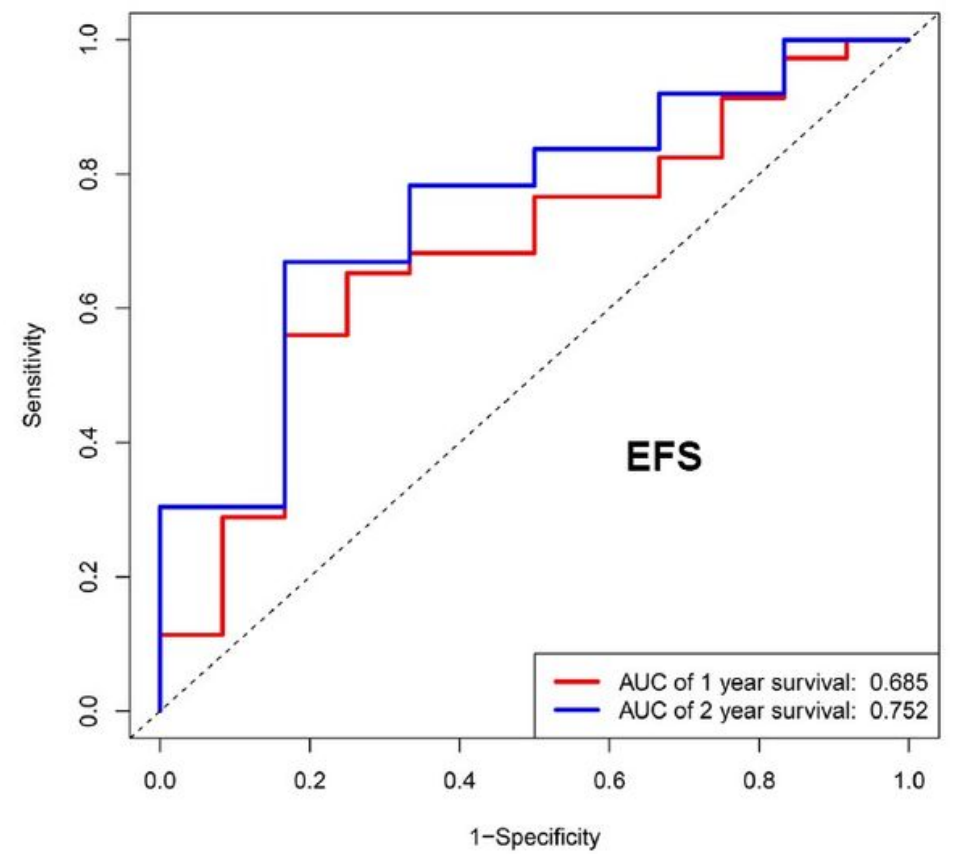

b

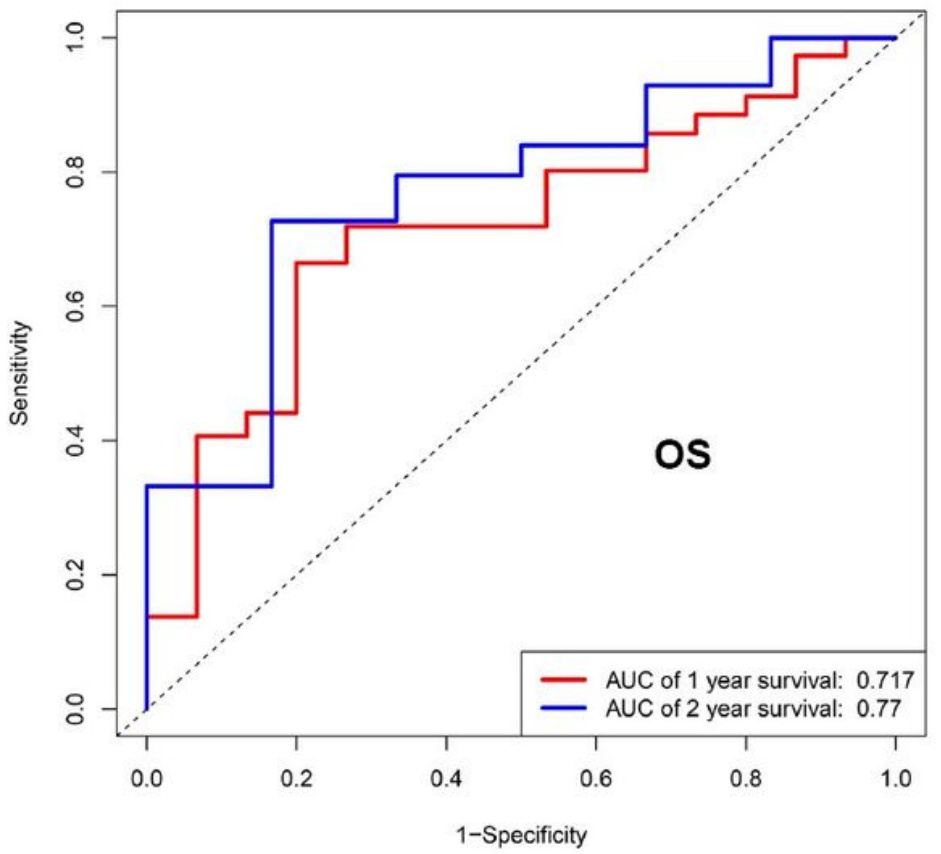

Figure 3

Area under the receiver operating characteristic curve (ROC) analysis of CD52 gene expression of CN-AML patients in TCGA dataset. a: ROC curves for EFS; b: ROC curves for OS. 


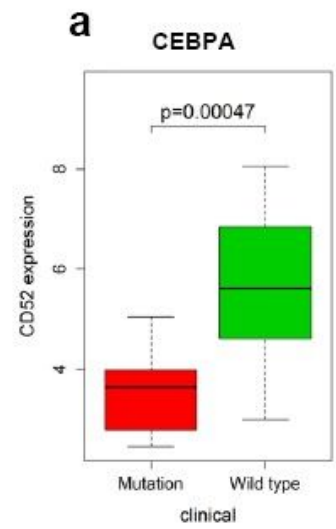

e

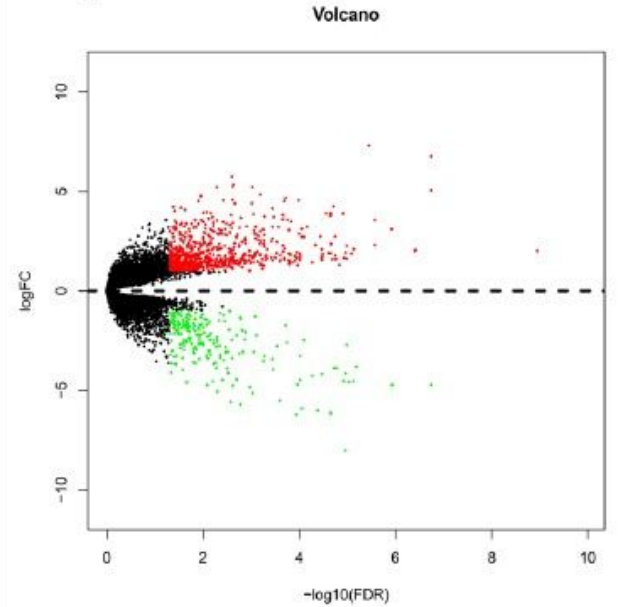

C
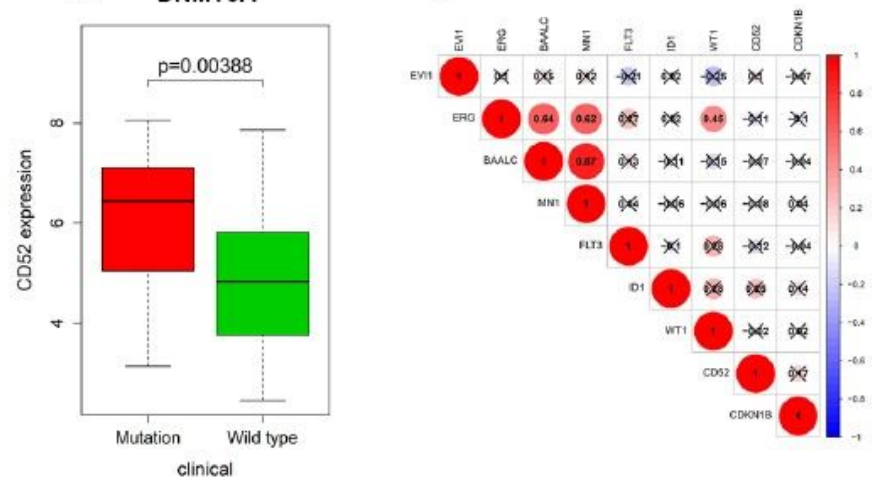

f

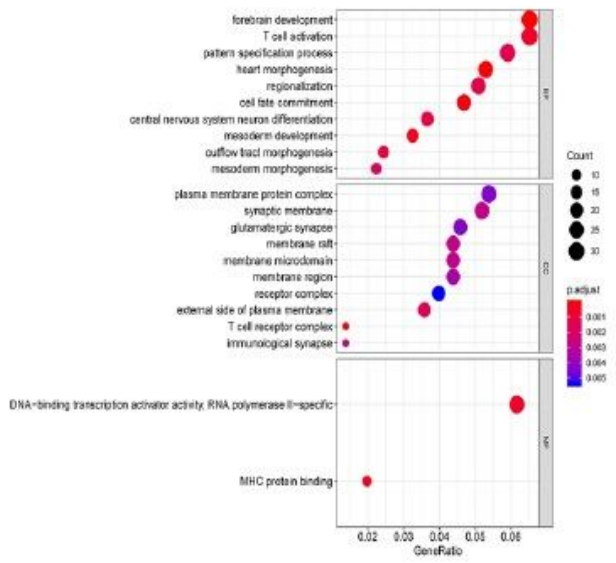

d

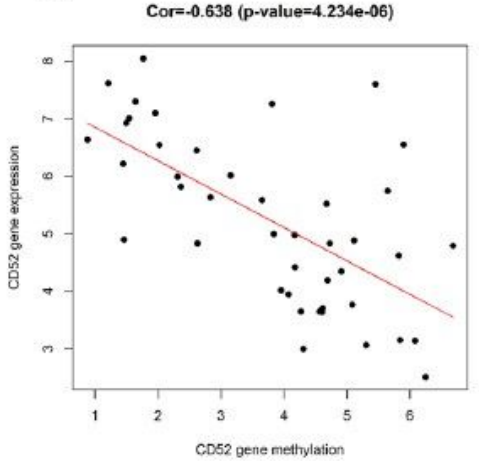

g

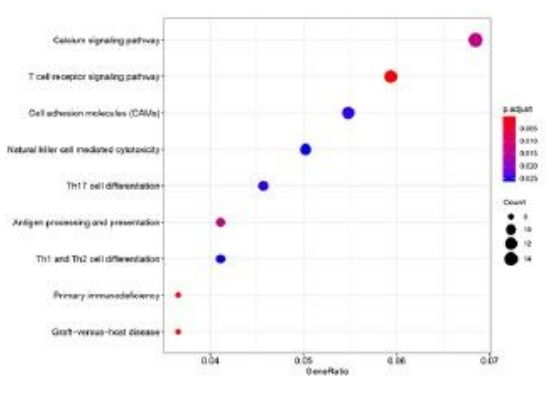

Figure 4

Molecular signatures associated with CD52 in CN-AML from TCGA cohort. a-b: The relationship between CD52 expression and gene mutation. C: Correlation between gene (WT1, EVI1, FLT3, MN1, ERG, ID1, CDKN1B, CD52) expression. The color indicates the $P$ value and $P$ value $<0.05$ is considered statistically significant. d: Correlation between mRNA expression and DNA methylation of CD52. e: Volcano plot of differentially expressed genes (DEGs). f-g: Enrichment analysis of GO and KEGG pathway of DEGs. The size of the dots represents the number of core genes, and the color indicates the adjust $\mathrm{p}$. (only show the top 10 enrichment pathways).

\section{Supplementary Files}

This is a list of supplementary files associated with this preprint. Click to download.

- Additionalfile1.pdf 